\title{
Current concepts in the management of hepatopulmonary syndrome
}

Vincent Ho

School of Medicine, James Cook University, Cairns, Queensland,

Australia
Correspondence: Vincent Ho School of Medicine, James Cook University, Cairns,

Queensland 4870, Australia

$\mathrm{Tel}+$ I 61740506333

Fax + | 61 $7403 \mid$ II68

Email vincent_ho@health.qld.gov.au

\begin{abstract}
The hepatopulmonary syndrome is characterized as the triad of liver disease, pulmonary gas exchange abnormalities leading to arterial deoxygenation and evidence of intrapulmonary vascular dilatations. This review summarizes the pathological mechanisms leading to pulmonary vascular changes in hepatopulmonary syndrome. The role of the three currently used diagnostic imaging modalities of contrast-enhanced echocardiography, perfusion lung scanning and pulmonary arteriography that identify the presence of intrapulmonary vascular abnormalities are reviewed. Liver transplantation is considered to be the definitive treatment of hepatopulmonary syndrome with often successful reversal of hypoxemia, however other treatments have been trialed. This review further appraises the evidence for the use of pharmacological agents and the role of radiological interventions in hepatopulmonary syndrome.
\end{abstract}

Keywords: hepatopulmonary syndrome, liver cirrhosis, lung diseases

\section{Hepatopulmonary syndrome}

Hepatopulmonary syndrome (HPS) is defined as the triad of liver disease, pulmonary gas exchange abnormalities leading to arterial deoxygenation, and evidence of intrapulmonary vascular dilatations (Krowka 2000). The prevalence in the setting of cirrhosis varies from 4\% (Stoller et al 1995) to 47\% (Hopkins et al 1992). This disparity in prevalence rates can be attributed to different cut-offs in defining arterial hypoxemia in primary studies. HPS is a pulmonary vascular disorder responsible for an increased morbidity and mortality among patients awaiting liver transplantation (Swanson et al 2005).

A relationship between the liver and the lung was first noted by Fluckiger (1884) based on his observation of a woman with cirrhosis, cyanosis, and clubbed digits. This relationship was not formalized until almost a century later when Kennedy and Knudson (1997) described 'hepatopulmonary syndrome' as a clinicopathological entity characterized by hypoxemia and the pathogenetic hallmark of intrapulmonary vascular dilatations.

Severe hepatic dysfunction is not required and HPS can present in patients with mild liver disease (Abrams et al 1995).

This article reviews the clinical features, diagnostic modalities as well as current concepts regarding the pathogenesis of HPS. The evidence base to support pharmacological and nonpharmacological therapies is discussed.

\section{Clinical manifestations}

The clinical features of HPS are typically pulmonary manifestations. Dyspnea is the most common complaint and can be insidious in presentation. Platypnea defined as dyspnea exacerbated by sitting up and improved with lying down is a clinical feature of HPS. Orthodeoxia, defined as arterial deoxygenation accentuated in the upright position versus the supine position often accompanies platypnea and is highly specific 
for HPS in the context of chronic liver disease (Seward et al 1984). The cutoff value for orthodeoxia is delineated as a $\mathrm{PaO}_{2}$ decrease of $5 \%$ or more, or $4 \mathrm{~mm} \mathrm{Hg}$ or more from the supine to upright position (Rolla et al 1997). The mechanism for orthodeoxia is attributed to preferential perfusion of the lung bases so that functional shunting is greater when the patient is upright (Robin et al 1976). Most patients will have stigmata of chronic liver disease on physical examination.

Alizadeh and colleagues (2006) reviewed 54 cirrhotic patients of which $10(18.5 \%)$ and 7 cases (13\%) had clinical and subclinical features of HPS, respectively. Dyspnea was a presenting feature of all patients with HPS and cyanosis was present in $90 \%$ of all cases. The presence of clubbing had the highest positive predictive value (75\%) and dyspnea the highest negative predictive value (100\%) for HPS.

Spider nevi are another common clinical feature of patients with HPS (Andrivet et al 1993; Lima et al 2004). Rodriguez-Roisin and colleagues (1987) noted that there was a significant relationship between cutaneous spider angiomata and systemic and pulmonary vasodilatation suggesting that spider nevi may well represent a cutaneous marker for intrapulmonary vascular dilatations.

\section{Natural history}

The prognosis associated with HPS is poor. A prospective study of 27 patients with HPS showed that the presence of HPS is a major independent risk factor on the survival of patients with cirrhosis (Schenk et al 2003). In this study the median survival time in cirrhotic patients with HPS was 10.6 months compared to 40.8 months in cirrhotic patients without HPS. At an observation period of 2.5 years the mortality rate for HPS patients was approximately $63 \%$. The leading cause of death was hemorrhagic shock secondary to gastrointestinal bleeding.

In a retrospective study of 22 patients with HPS the mortality rate was $41 \%$ after a mean time of 2.5 years after diagnosis (Krowka et al 1993).

The degree of arterial hypoxemia appears to influence survival. Swanson and colleagues (2005) demonstrated in a case-control study involving 61 HPS patients that long-term survival for HPS patients is worse in the subgroup with lower baseline $\mathrm{PaO}_{2}(\leq 50 \mathrm{mmHg})$.

\section{Pathogenesis}

The pathogenesis of HPS remains unknown. The hallmark of HPS is microvascular dilatation within the pulmonary arterial circulation. Microvascular dilatation impairs ventilation-perfusion matching and can produce anatomical and functional shunt physiology, leading to hypoxemia.
Early on some investigators believed that functional vasodilatation of the pulmonary vasculature was attributable to inadequate synthesis or metabolism of pulmonary vasoactive substances by a damaged liver (Bruix et al 1985; Krowka and Cortese 1990; Pizcueta et al 1992).

In the quest to define the nature of these causal vasoactive substances, the potent vasodilator nitric oxide was identified as a leading agent. Exhaled nitric oxide levels (a measure of pulmonary production) increase in cirrhotic patients with HPS and normalize after orthotopic liver transplantation (Cremona et al 1995; Rolla et al 1997). Overexpression of both endothelial nitric oxide synthase and inducible nitric oxide synthase are found in the pulmonary microcirculation (Fallon et al 1997; Nunes et al 2001).

Moreover one case report has revealed that inhibition of nitric oxide production with NG-LNAME transiently improves HPS with improvements in exercise tolerance and $\mathrm{PaO}_{2}$ (Brussino et al 2003). Methylene blue - a dye that inhibits the effects of nitric oxide on soluble guanylate cyclase - was administered by a single intravenous dose in a small study of 7 patients with HPS, established cirrhosis and a hyperdynamic circulatory state (Schenk et al 2000). These patients experienced significant improvements in gas exchange accompanied by an increase in pulmonary vascular resistance.

Intestinal endotoxemia with accompanying nitric oxide release and tumor necrosis factor (TNF)- $\alpha$ overproduction, secondary to enteral translocation of Gram-negative bacteria due to portal hypertension has been proposed as a cause of excessive pulmonary vasodilatation (Zhang et al 2005). Improvements in arterial oxygenation have been recorded in HPS patients receiving antibiotic treatment (Rabiller et al 2002). Pentoxifylline, a nonspecific phosphodiesterase inhibitor that is recognized to block effects mediated by TNF- $\alpha$ in inflammatory and endothelial cells, has been shown to markedly improve intrapulmonary vasodilatation and gas exchange abnormalities in rats without altering systemic or portal pressures (Zhang et al 2007).

Other studies have looked at the contribution of vasoactive intestinal peptide, substance $P$, and atrial natriuretic factor as etiological agents in the process of pulmonary microvascular dilatation in cirrhosis (Henriksen et al 1980; Hortnagl et al 1984; Gines et al 1988).

Vasodilatation alone however is not thought to be responsible for the pathobiological process in HPS. A recent study found that while administration of inhaled L-NAME in ten HPS patients did attenuate systemic and pulmonary vasodilatation, V/Q mismatch and arterial hypoxemia did not improve (Gomez et al 2006). 
This study goes on to suggest that arterial deoxygenation in HPS is unlikely to be due to an ongoing vasodilator effect from enhanced pulmonary nitric oxide production.

Indeed vasodilatation alone is unlikely to account for the more than 10-fold increase in capillary diameters in HPS especially as there is little smooth muscle to relax in normal capillaries (Naeijie and Dinh-Xuan 2005).

A new concept has emerged that the pulmonary microcirculation in patients with cirrhosis may manifest through decreased hypoxic pulmonary vascular constriction accompanied by a vasoconstrictive release leading to dilatation (Naeijie et al 1981, Gomez et al 2006). A key goal of research in HPS is to establish how such pulmonary vascular changes relate to the systemic vasodilatation which underlies the hyperdynamic circulatory and neuroregulatory dysfunction state seen in cirrhotic patients.

\section{Mechanisms of hypoxemia}

Intrapulmonary vascular dilatations especially in alveolar regions, are the defining pathological hallmark of HPS and are thought to be responsible for hypoxemia (Davis et al 1978; Edell et al 1989). Rydell and Hoffbauer (1956) first documented the presence of numerous intrapulmonary arteriovenous anastomoses in their postmortem report of a patient with juvenile cirrhosis and cyanosis. This finding in cirrhotic patients was confirmed radiologically through injections of micro-opaque gelatin to show widespread dilation of the pulmonary vasculature at precapillary and capillary levels (Berthelot et al 1966).

The normal diameter of capillaries in the alveolar septum is $7-15$ microns (Davis et al 1978) and particles larger than 15 microns are normally trapped in the pulmonary vasculature. Several studies used intravenous radiolabeled macroaggregated albumin particles larger than 20 microns in diameter to establish the presence of dilated pulmonary microvasculature as these particles were shown to traverse the pulmonary vasculature and enter systemic circulation (Robin et al 1975; Genovesi et al 1976; Wolfe et al 1977). Intrapulmonary capillary dilatations as large as 500 microns in diameter have been found (Williams et al 1979).

In a true anatomical shunt hypoxemia persists despite $100 \%$ oxygen inhalation. Supplementary oxygen however does improve oxygenation in HPS and is a recommended treatment for HPS. A hypothesis has been developed to account for the mechanisms of hypoxemia in HPS known as the alveolar-capillary oxygen disequilibrium (Davis et al 1978). This hypothesis proposes that when the capillary is grossly dilated, oxygen molecules from adjacent alveoli are unable to permeate sufficiently to the center of the dilated vessel. It is the center of the vessel that tends to contain the stream of erythrocytes carrying hemoglobin and thus inadequate oxygenation of hemoglobin leads to systemic hypoxemia. This diffusion defect is able to be partially overcome by the use of supplementary oxygen.

It is now evident that intrapulmonary vascular dilatations can occur in one of two patterns (Krowka and Cortese 1990). The more common Type I lesions are characterized by diffuse pulmonary vascular dilatations at the precapillary level close to the normal gas exchange units of the lung whereas Type II lesions are more discrete, localized dilatations and are actually large arteriovenous communications distant from the gas exchange units. Supplementary oxygen improves Type I HPS $\mathrm{PaO}_{2}$ but Type 2 HPS has a poor response to oxygen as true anatomical shunting is present.

The fraction of total cardiac output conducted by intrapulmonary vascular dilations can be as high as 70\% (Wolfe et al 1977). This increased cardiac output reduces transit time through the low-resistance pulmonary vasculature and a decrease in intrapulmonary transit time contributes to hypoxemia. Exercising patients with HPS can shorten transit time and worsen oxygenation through the formation of a greater functional shunt (Thorens and Junod 1992).

\section{Diagnosis}

In the evaluation of the hypoxemic cirrhotic patient the exclusion of other contributing cardiopulmonary causes such as pulmonary atelectasis, ascites, chronic obstructive pulmonary disease, and hepatic hydrothorax is mandatory. A careful history and physical examination aids in the consideration of alternative diagnoses of dyspnea.

An arterial blood gas analysis to detect hypoxemia is the next investigation of choice. Schenk and colleagues (2002) noted the difficulty of setting arbitrary cut-off values for hypoxemia in published studies. The use of different cut-off values defining arterial hypoxemia in studies led to a wide range of prevalence rates for HPS in patients with cirrhosis. Their study showed that arterial hypoxemia defined as a $\mathrm{PaO}_{2}<70 \mathrm{mmHg}$ or below the age-related threshold predicted the presence of HPS with high probability in the absence of intrinsic cardiopulmonary diseases.

If gas exchange abnormalities are detected chest radiography and pulmonary function tests can be used to help rule out other cardiopulmonary abnormalities.

However, even in the presence of chronic cardiopulmonary conditions the diagnosis of HPS can be made if there is evidence of pulmonary vasodilatation with demonstrable 
functional shunting. This can be achieved by means of contrast echocardiography using intravenous injections of agitated saline or indocyanine green to produced bubbles of at least 15 microns in diameter (Kamath 2002). Under normal circumstances these microbubbles are trapped in the pulmonary vasculature and absorbed. However in the presence of intracardiac right to left shunts, these microbubbles are seen in the left heart within the first three cardiac cycles (Shub et al 1976). In hepatopulmonary syndrome, the bubbles are seen in the left heart after the third heart beat, usually between the third and sixth heart beat.

Much work has focused on echocardiographic examination in the evaluation of HPS. For instance a measured maximal left atrial volume $\geq 50 \mathrm{ml}$ has been shown recently to be a simple and feasible parameter to detect HPS in cirrhotic patients (Zamirian et al 2007). Right ventricular diastolic dysfunction is notably more common in cirrhotic patients with HPS than cirrhotic patients without HPS (Karabulut et al 2006). Vedrinne and colleagues (1997) found that transesophageal echocardiography using gelatin contrast solution to be a more sensitive test than transthoracic echocardiography with an intrapulmonary shunt detection rate of $51 \%$ compared to $32 \%$.

There are however a number of limitations of contrastenhanced echocardiography (Wang and Lin 2005). Most notably contrast-enhanced echocardiography cannot quantify the degree of intrapulmonary shunting. It is also unable to differentiate between intrapulmonary vascular dilatation and direct arteriovenous communication. Finally specificity is lacking as there are cirrhotic patients with a positive echocardiographic study that have normal arterial blood gas profiles and thus do not meet the definition of HPS (Hopkins et al 1992).

${ }^{99}$ Technetium macroaggregated albumin perfusion lung scanning overcomes the first limitation of contrast echocardiography as it permits quantification of the degree of intrapulmonary shunting (Abrams et al 1998). In perfusion lung scanning albumin macroaggregates $>20$ microns in diameter are normally entrapped in pulmonary vasculature but in patients with intracardiac/intrapulmonary shunts these particles are able to enter systemic circulation and are taken by other organs in the body. Normally, less than $5 \%$ of the isotope can be quantitated over the brain. In hepatopulmonary syndrome, there is $>6 \%$ uptake in the brain. The major disadvantage of the ${ }^{99} \mathrm{Technetium}$ macroaggregated albumin scan is that it cannot differentiate intracardiac from intrapulmonary shunting.

Contrast enhanced cardiography has been shown to be more sensitive than lung perfusion scanning for the detection of intrapulmonary vascular dilatations (Abrams et al 1995). It has the additional advantage in that it can be performed as part of routine echocardiographic screening for pulmonary hypertension and the European Respiratory Society Task Force on Pulmonary-Hepatic vascular disorders has recommended contrast-enhanced echocardiography as the first line screening modality for HPS (Rodriguez-Roisin et al 2004).

Pulmonary angiography is reserved for those patients who have a poor response to $100 \%$ oxygen, demonstrated by an increase in the $\mathrm{PaO}_{2}$ to less than $300 \mathrm{mmHg}$ (Kamath 2002). It is an invasive procedure that can delineate the appearance of the pulmonary vasculature. There are 2 patterns seen with pulmonary angiography: Type 1 HPS as characterized by precapillary pulmonary artery dilatation without arteriovenous fistulas and Type 2 HPS where localized pulmonary arteriovenous fistulous communications are seen (Scott et al 1999; Ryu and Oh 2003). Type 1 angiographic findings can vary from normal to an advanced diffuse 'spider-like' or spongy appearance (Krowka et al 1993; Castro and Krowka 1996). These Type 1 HPS patients with advanced diffuse pulmonary changes have more severe hypoxemia and respond poorly to $100 \%$ oxygen (Krowka et al 1993).

Type 2 HPS which is less common, does not respond to $100 \%$ oxygen with partial pressure measurements generally not exceeding $200 \mathrm{~mm} \mathrm{Hg}$ with 100\% oxygen (Scott et al 1999). Patients with Type 2 HPS should be considered for embolotherapy although there are case reports of coil embolization in Type 1 HPS used as a temporizing measure prior to liver transplantation (Ryu and Oh 2003; Saad et al 2007).

Remodeling of the pulmonary vasculature does occur after liver transplantation although the time taken for this to occur is variable, in some cases over a year (Fallon and Abrams 2000).

\section{Management}

Many pharmacological treatments have been trialed in the management of HPS. Ostensibly such treatments target the putative mediators of HPS such as nitric oxide and TNF- $\alpha$ although quite often pharmacological agents are tried with no substantive physiological basis.

Nitric oxide antagonists such as NG-nitro-L-argininemethylester (L-NAME) and methylene blue as described previously have been trialed with variable improvements in gas exchange (Schenck et al 2000; Brussino et al 2003). Somatostatin analogues, cyclooxygenase inhibitors, and immunosuppressive agents such as glucocorticoids and cyclophosphamide have been trialed with mixed and more 
often disappointing results (Shijo et al 1991; Krowka et al 1993; Soderman et al 1994; Cadranel et al 1992).

Almitrine bimesylate a selective pulmonary vasoconstrictor was thought to be a promising agent for HPS as in theory via its vasoconstrictive effect it may lower physiological shunting and thereby reduce existing diffusion defects. It has had notable success in isolated case reports (Milhe et al 2006) however Krowka and Cortese (1987) found that only 1 of the 5 patients receiving almitrine bismesylate in their pilot study had any improvement in oxygenation and that the magnitude of this response was small $(<10 \mathrm{~mm} \mathrm{Hg}$ increase in $\mathrm{PaO}_{2}$ on breathing room air). In another study almitrine bismesylate failed to produce a significant increase in $\mathrm{PaO}_{2}$ in 6 patients with HPS (Nakos et al 1993).

Interestingly, the use of garlic powder capsules daily for a minimum of 6 months resulted in a modest improvement in arterial oxygenation in 6 out of 15 subjects in a small pilot trial (Abrams and Fallon 1998).

Antibiotic treatment has resulted in improvements in arterial oxygenation in HPS patients (Rabiller et al 2002). The nonspecific phosphodiesterase inhibitor pentoxifylline that is a recognized TNF- $\alpha$ blocker has been shown to improve intrapulmonary vasodilatation and gas exchange abnormalities, but this is demonstrable only in animal studies (Zhang et al 2007). A phase 1 open-label pilot study of pentoxifylline in humans with HPS was recently terminated because of poor tolerability and substantial side effects (NIH 2008).

A case report of the vasodilator Iloprost describes a benefit in the reduction of hypoxia, dyspnea and an increase in exercise capacity mediated possibly through widespread dilation of pulmonary capillaries with ensuing decreased pulmonary shunting and increased transit time of erythryocytes (Krug et al 2007).

The limitation with the evidence base to date for pharmacological treatment in HPS is that data is presented through either case reports or small, uncontrolled trials that lack sufficient size to test for efficacy. Randomized controlled trials with sufficient sample sizes are required to investigate new pharmacological interventions.

The development of portal hypertension is thought to play a key role in the pathogenesis of HPS. A reduction in portal pressure appears to be beneficial in HPS and a few case reports (Allgaier et al 1995; Riegler et al 1995; Selim et al 1998) have documented variable improvements in gas exchange with the use of transjugular intrahepatic portosystemic shunts (TIPS). However a more recent study involving 3 patients with HPS the use of TIPS did not lead to any overall sustained change in arterial blood gas profile
(Martinez-Palli et al 2005) and there is currently insufficient evidence to support TIPS as a recommended treatment in HPS.

Other radiological interventional procedures have been trialed in HPS. Percutaneous transluminal balloon cavaloplasty is an effective treatment in patients with suprahepatic inferior vena cava (IVC) obstruction (Yamada et al 1983). HPS sometimes occurs in cases of IVC obstruction and in one report a patient with HPS and IVC obstruction after undergoing balloon cavaloplasty experienced significant improvement in dyspnea, cyanosis, and hypoxemia, with intrapulmonary shunt reversal (De et al 2000). In a followup study, De and colleagues (2002) showed an impressive intrapulmonary shunt reversal in 5 out of 6 cases.

Type 2 HPS as noted previously is characterized by pulmonary arteriovenous fistulas and does not respond well to oxygen therapy. Transcatheter coil embolization of the arteriovenous pulmonary fistulas in type 2 HPS has been successful in reducing morbidity of HPS before and after liver transplantation (White et al 1988; Poterucha et al 1995). Embolotherapy may be a reasonable first line of bridging therapy in patients with Type 2 HPS prior to transplantation. Even in Type 1 patients with advanced diffuse pulmonary changes, benefits in reducing morbidity pre-transplantation have been described with coil embolization (Ryu and Oh 2003; Saad et al 2007).

An important therapy for HPS is oxygen supplementation which does lead to significant improvements in hypoxemia. Long-term supplementary oxygenation thus is advised in all hypoxemic HPS patients despite the lack of data to show improvements in survival.

The only definitive treatment for HPS is orthotopic liver transplantation. The data to support this is incontrovertible although the mechanism of how the pulmonary vasculature is remodeled after transplantation is not clearly understood. What is known is that at least $85 \%$ of all cases of patients with HPS undergoing liver transplantation experience either significant improvement or complete resolution in hypoxemia (Lange and Stoller 1996). This resolution however may take time and in some cases over a year (Fallon and Abrams 2000). Unfortunately the mortality after liver transplantation is significantly increased in HPS patients with a 1-year survival rate of $71 \%$ noted in one cohort study (Arguedas et al 2003). Survival is worse for those patients with more severe hypoxemia and significant intrapulmonary shunting.

The understanding that orthotopic liver transplantation provides the best and often complete management of HPS has revolutionized the therapeutic approach to this unique 
disease entity. No longer is hypoxemia in such patients considered a relative contraindication for transplantation. In fact the degree of hypoxemia is considered critical in the consideration for liver transplantation. The ERS Task Force on Pulmonary-Hepatic Vascular Disorders recommends as a firm indication for orthotopic liver transplantation if $\mathrm{PaO}_{2} \geq 50-<60 \mathrm{mmHg}$ and consideration of orthotopic liver transplantation on an individual basis if $\mathrm{PaO}_{2}<50 \mathrm{mmHg}$ (Rodriguez-Roisin et al 2004).

As no other accepted medical therapy exists at the present time for HPS other than supplementary oxygen, proceeding with orthotopic liver transplantation in suitable HPS patients is the best step forward.

\section{Disclosure}

The author reports no conflict of interest in this work.

\section{References}

Abrams GA, Jaffe CC, Hoffer PB, et al. 1995. Diagnostic utility of contrast echocardiography and lung perfusion scan in patients with hepatopulmonary syndrome. Gastroenterology, 109:1283-8.

Abrams GA, Nanda NC, Dubovsky EV, et al. 1998. The use of macroaggregated albumin lung perfusion scan to diagnose hepatopulmonary syndrome: a new approach. Gastroenterology, 114:305-10.

Abrams GA, Fallon MB. 1998. Treatment of hepatopulmonary syndrome with Allium sativum L. (garlic): a pilot trial. J Clin Gastroenterol, 27:232-5.

Alizadeh AHM, Fatemi SR, Mirzaee V, et al. 2006. Clinical features of hepatopulmonary syndrome in cirrhotic patients. World $J$ Gastroenterol, 12:1954-6.

Allgaier HP, Haag K, Ochs A, et al. 1995. Hepatopulmonary syndrome: successful treatment by transjugular intrahepatic portosystemic stentshunt (TIPS). J Hepatol, 23:102-5.

Andrivet P, Cadranel J, Housset B, et al. 1993. Mechanisms of impaired arterial oxygenation in patients with liver cirrhosis and severe respiratory insufficiency. Effects of indomethacin. Chest, 103:500-7.

Arguedas M, Abrams GA, Krowka MJ, et al. 2003. Prospective evaluation of outcomes and predictors of mortality in patients with hepatopulmonary syndrome undergoing liver transplantation. Hepatology, 37:192-7.

Berthelot P, Walker JG, Sherlock S, et al. 1966. Arterial changes in the lungs in cirrhosis of the liver - lung spider nevi. $N$ Engl $J$ Med, 274:291-8.

Bruix J, Bosch J, Kravetz D, et al. 1985. Effects of prostaglandin inhibition on systemic and hepatic hemodynamics in patients with cirrhosis of the liver. Gastroenterology, 88:430-5.

Brussino L, Bucca C, Morello M, et al. 2003. Effect on dyspnoea and hypoxemia of inhaled NG-nitro-L-arginine methyl ester in hepatopulmonary syndrome. Lancet, 362:43-4.

Cadranel J, Milleron B, Cadranel JF, et al. 1992. Severe hypoxemia associated intrapulmonary shunt in a patient with chronic liver disease: improvement after medical treatment. Am Rev Respir Dis, 146:526-7.

Castro M, Krowka MJ. 1996. Hepatopulmonary syndrome: a pulmonary vascular complication of liver disease. Clin Chest Med, 17:35-48.

Cremona G, Higenbottam TW, Mayoral V, et al. 1995. Elevated exhaled nitric oxide in patients with hepatopulmonary syndrome. Eur Respir J, 8:1883-5.

Davis HH 2nd, Schwartz DJ, Lefrak SS, et al. 1978. Alveolar-capillary oxygen disequilibrium in hepatic cirrhosis. Chest, 73:507-11.
De BK, Sen S, Biswas PK, et al. 2000. Hepatopulmonary syndrome in inferior vena cava obstruction responding to cavoplasty. Gastroenterology, 118:192-6.

De BK, Sen S, Biswas PK, et al. 2002. Occurrence of hepatopulmonary syndrome in Budd-Chiari syndrome and the role of venous decompression. Gastroenterology, 122:897-903.

Edell ES, Cortese DA, Krowka MJ, et al. 1989. Severe hypoxemia and liver disease. Am Rev Respir Dis, 140:1631-5.

Fallon MB, Abrams GA, Luo B, et al. 1997. The role of endothelial nitric oxide synthase in the pathogenesis of a rat model of hepatopulmonary syndrome. Gastroenterology, 113:606-14.

Fallon MB, Abrams GA. 2000. Pulmonary dysfunction in chronic liver disease. Hepatology, 32:859-65.

Fluckiger M. 1884. Vorkommen von trommelschlägelförmigen fingerendphalengen ohne chronische veränderungen an der lungen oder am herzen. Wien Med Wschr, 34:1457.

Genovesi MG, Tierney DF, Taplin GV, et al. 1976. An intravenous radionuclide method to evaluate hypoxemia caused by abnormal alveolar vessels. Limitation of conventional techniques. Am Rev Respir Dis, 114:59-65.

Gines P, Jimenez W, Arroyo V, et al. 1988. Atrial natriuretic factor in cirrhosis with ascites: Plasma levels, cardiac release and splanchnic extraction. Hepatology, 8:636-42.

Henriksen JH, Staun-Olsen P, Fahrenkrug P, et al. 1980. Vasoactive intestinal polypeptide (VIP) in cirrhosis: arteriovenous extraction in different vascular beds. Scand J Gastroenterol, 15:787-92.

Hopkins WE, Waggoner AD, Barzilai B. 1992. Frequency and significance of intrapulmonary right-to-left shunting in end-stage hepatic disease. Am J Cardiol, 70:516-19.

Hortnagl H, Singer EA, Lenz K, et al. 1984. Substance-P is markedly increased in plasma of patients with hepatic coma. Lancet, 1:480-3.

Kamath PS. 2002. Portopulmonary hypertension and hepatopulmonary syndrome. J Gastroenterol Hepatol, 17:S253-5.

Karabulut A, Iltumur K, Yalcin K, et al. 2006. Hepatopulmonary syndrome and right ventricular diastolic functions: an echocardiographic examination. Echocardiography, 23:271-8.

Kennedy TC, Knudson RJ. 1997. Exercise-aggravated hypoxemia and orthodexia in cirrhosis. Chest, 72:305-9.

Krowka MJ, Cortese DA. 1987. Severe hypoxemia associated with liver disease: Mayo Clinic experience and the experimental use of almitrine bimesylate. Mayo Clin Proc, 62:164-73.

Krowka MJ, Cortese DA. 1990. Hepatopulmonary syndrome: an evolving perspective in the era of liver transplantation. Hepatology, 11:138-42.

Krowka MJ, Dickson ER, Cortese DA. 1993. Hepatopulmonary syndrome. Clinical observations and lack of therapeutic response to somatostatin analogue. Chest, 104:515-21.

Krowka MJ. 2000. Hepatopulmonary syndromes. Gut, 40:1-4.

Krug S, Seyfarth HJ, Hagendorff A, et al. 2007. Inhaled iloprost for hepatopulmonary syndrome: improvement of hypoxemia. Eur J Gastroenterol Hepatol, 19:1140-3.

Lange PA, Stoller JK. 1996. Hepatopulmonary syndrome: effects of liver transplantation. Clin Chest Med, 17:115-23.

Lima BL, Franca AV, Pazin-Filho, et al. 2004. Frequency, clinical characteristics, and respiratory parameters of hepatopulmonary syndrome. Mayo Clin Proc, 79:42-8.

Martinez-Palli G, Drake BB, Garcia-Pagan JC, et al. 2005. Effect of transjugular intrahepatic portosystemic shunt on pulmonary gas exchange in patients with portal hypertension and hepatopulmonary syndrome. World J Gastroenterol, 11:6858-62.

Milhe F, Reynaud-Gaubert M, Magnan A, et al. 2006. Oxygenation improvement with almitrine bismesylate in the hepatopulmonary syndrome. Respiratory Medicine Extra, 2:81-4.

Naejie R, Hallemans R, Mols P, et al. 1981. Hypoxic pulmonary vasoconstriction in liver cirrhosis. Chest, 80:570-4.

Naejie R, Dinh-Xuan AT. 2005. The hepatopulmonary syndrome: NO way out? Eur Respir J, 25:212. 
Nakos G, Evrenoglou D, Vassilakis N, et al. 1993. Haemodynamics and gas exchange in liver cirrhosis: the effect of orally administered almitrine bimesylate. Respir Med, 87:93-8.

[NIH] National Institutes of Health. 2008. Pilot study of pentoxifylline for hepatopulmonary syndrome [online]: identifier: NCT00593658. Accessed on June 25, 2000. URL: http://clinicaltrials.gov/ct2/show/ NCT00593658?term $=$ NCT00593658\&rank $=1$.

Nunes H, Lebrec D, Mazmanian M, et al. 2001. Role of nitric oxide in hepatopulmonary syndrome in cirrhotic rats. Am J Respir Crit Care Med, 164:879-85.

Pizcueta P, Pique JM, Fernandez M, et al. 1992. Modulation of the hyperdynamic circulation of cirrhotic rats by nitric oxide inhibition. Gastroenterology, 103:1909-15.

Poterucha JJ, Krowka MJ, Dickson ER, et al. 1995. Failure of hepatopulmonary syndrome to resolve after liver transplantation and successful treatment with embolotherapy. Hepatology, 21:96-100.

Rabiller A, Nunes H, Lebrec D, et al. 2002. Prevention of gram-negative translocation reduces the severity of hepatopulmonary syndrome. Am $J$ Respir Crit Care Med, 166:514-17.

Riegler JL, Lang KA, Johnson SP, et al. 1995. Transjugular intrahepatic portosystemic shunt improves oxygenation in hepatopulmonary syndrome. Gastroenterology, 109:978-83.

Robin ED, Horn B, Goris ML, et al. 1975. Detection, quantitation and pathophysiology of lung spiders. Trans Assoc Am Physicians, 88:202-16.

Robin ED, Laman D, Horn BR, et al. 1976. Platypnea related to orthodeoxia caused by true vascular lung shunts. $N$ Engl J Med, 294:941-3.

Rodriguez-Roisin R, Roca J, Agusti AG, et al. 1987. Gas exchange and pulmonary vascular reactivity in patients with liver cirrhosis. Am Rev Respir Dis, 135:1085-92.

Rodriguez-Roisin R, Krowka MJ, Herve P, et al. 2004. Pulmonary-Hepatic vascular Disorders (PHD). Eur Respir J, 24:861-80.

Rolla G, Brussino L, Colagrande P, et al. 1997. Exhaled nitric oxide and oxygenation abnormalities in hepatic cirrhosis. Hepatology, 26:842-7.

Rydell R, Hoffbauer FW. 1956. Multiple pulmonary arteriovenous fistulas in juvenile cirrhosis. Am J Med, 21:450-60.

Ryu JK, Oh JH. 2003. Hepatopulmonary syndrome: angiography and therapeutic embolization. Clin Imaging, 27:97-100.

Saad NEA, Lee DE, Waldman DL et al. 2007. Pulmonary arterial coil embolization for the management of persistent Type 1 hepatopulmonary syndrome after liver transplantation. J Vasc Interv Radiol, 18:1576-80.

Schenk P, Madl C, Rezaie-Majid S, et al. 2000. Methylene blue improves the hepatopulmonary syndrome. Ann Intern Med, 133:701-6.

Schenk P, Fuhrmann V, Madl C, et al. 2002. Hepatopulmonary syndrome: prevalence and predictive value of various cut offs for arterial oxygenation and their clinical consequences. Gut, 51:853-9.

Schenk P, Schöniger-Hekele M, Fuhrmann V, et al. 2003. Prognostic significance of the hepatopulmonary syndrome in patients with cirrhosis Gastroenterology, 125:1042-52.

Scott VL, Dodson SF, Kang Y. 1999. The hepatopulmonary syndrome. Surg Clin North Am, 79:23-41.
Selim KM, Akriviadis EA, Zuckerman E, et al. 1998. Transjugular intrahepatic portosystemic shunt: a successful treatment for hepatopulmonary syndrome. J Am Coll Gastroenterol, 93:455-8.

Seward JB, Hayes DL, Smith HC, et al. 1984. Platypnea-orthodeoxia: clinical profile, diagnostic work-up, management and report of 7 cases. Mayo Clin Proc, 59:221.

Shijo H, Sasaki H, Yuh K, et al. 1991. Effects of indomethacin on hepatogenic pulmonary angiodysplasia. Chest, 99:1027-9.

Shub C, Tajik AJ, Seward JB, et al. 1976. Detecting intrapulmonary right-toleft shunt with contrast echocardiography: observations in a patient with diffuse pulmonary arteriovenous fistulas. Mayo Clin Proc, 51:81-4.

Soderman C, Juhlin-Dannfelt A, Lagerstrand L, et al. 1994. Ventilationperfusion relationships and central haemodynamics in patients with cirrhosis. Effects of a somatostatin analogue. J Hepatol, 21:52-7.

Stoller JK, Lange PA, Westveer MK, et al. 1995. Prevalence and reversibility of the hepatopulmonary syndrome after liver transplantation - The Cleveland Clinic Experience. West J Med, 163:133-8.

Swanson KL, Weiner RH, Krowka MJ. 2005. Natural history of hepatopulmonary syndrome: Impact of liver transplantation. Hepatology, 41:1122-29.

Thorens JB, Junod AF. 1992. Hypoxemia and liver cirrhosis: a new argument in favour of a diffusion-perfusion defect. Eur Respir $J$, 5:754-6.

Vedrinne JM, Duperret S, Bizollon T, et al. 1997. Comparison of transoesophageal and transthoracic contrast echocardiography for detection of an intrapulmonary shunt in liver disease. Chest, 111:1236-40.

Wang YW, Lin HC. 2005. Recent advances in hepatopulmonary syndrome. $J$ Chin Med Assoc, 68:500-5.

White RE, Lynch-Nyhan A, Terry P, et al. 1988. Pulmonary arteriovenous malformations: techniques and long-term outcome of embolotherapy. Radiology, 169:663-9.

Williams A, Trewby P, Williams R, et al. 1979. Structural alterations to the pulmonary circulation in fulminant hepatic failure. Thorax, 34:447-53.

Wolfe JD, Tashkin DP, Holly FE, et al. Hypoxemia of cirrhosis: detection of abnormal small pulmonary vascular channels by a quantitative radionuclide method. Am J Med, 63:746-54.

Yamada R, Sato M, Kawabata M, et al. 1983. Segmental obstruction of the hepatic inferior vena cava treated with transluminal angioplasty. Radiology, 149:91-6.

Zamirian M, Aslani A, Shahrzad S. 2007. Left atrial volume: A novel predictor of hepatopulmonary syndrome. Am J Gastroenterol, 102:1392-6.

Zhang HY, Han DW, Wang XG, et al. 2005. Experimental study on the role of endotoxin in the development of hepatopulmonary syndrome. World $J$ Gastroenterol, 11:567-72.

Zhang J, Ling Y, Tang L, et al. 2007. Pentoxifylline attenuation of experimental hepatopulmonary syndrome. J Appl Physiol, 102:949-55. 
